Bull. Korean Math. Soc. 51 (2014), No. 4, pp. 1023-1029

http://dx.doi.org/10.4134/BKMS.2014.51.4.1023

\title{
PARALLEL SECTIONS HOMOTHETY BODIES WITH MINIMAL MAHLER VOLUME IN $\mathbb{R}^{n}$
}

\author{
Youjiang Lin And Gangsong Leng
}

\begin{abstract}
In the paper, we define a class of convex bodies in $\mathbb{R}^{n}$-parallel sections homothety bodies, and for some special parallel sections homothety bodies, we prove that $n$-cubes have the minimal Mahler volume.
\end{abstract}

\section{Introduction}

The well-known Mahler's conjecture (see, e.g., [8], [15], [25] for references) states that, for any origin-symmetric convex body $K$ in $\mathbb{R}^{n}$,

$$
\mathcal{P}(K) \geq \mathcal{P}\left(C^{n}\right)=\frac{4^{n}}{n !}
$$

where $C^{n}$ is an $n$-cube and $\mathcal{P}(K)=\operatorname{Vol}(K) \operatorname{Vol}\left(K^{*}\right)$, which is known as the Mahler volume of $K$.

For $n=2$, Mahler [16] himself proved the conjecture, and in 1986 Reisner [22] showed that equality holds only for parallelograms. For $n=2$, a new proof of inequality (1.1) was obtained by Campi and Gronchi [4]. Recently, Lin and Leng [12] gave a new and intuitive proof of the inequality (1.1) in $\mathbb{R}^{2}$. Reisner (see, e.g., $[9,21,22]$ ) established the same inequality for a class of bodies that have a high degree of symmetry, known as zonoids. Inequality (1.1) was established by Saint Raymond [24] for bodies which are symmetric with respect to the coordinate hyperplanes. For the case of polytopes with at most $2 n+2$ vertices (or facets) (see, e.g., [2] for references), Lopez and Reisner [13] proved the inequality (1.1) for $n \leq 8$ and the minimal bodies are characterized. Recently, Nazarov, Petrov, Ryabogin and Zvavitch [20] proved that the cube is a strict local minimizer for the Mahler volume in the class of origin-symmetric convex bodies endowed with the Banach-Mazur distance.

Received August 6, 2013; Revised November 15, 2013.

2010 Mathematics Subject Classification. 52A10, 52A40.

Key words and phrases. convex body, polar body, parallel sections homothety bodies, Mahler conjecture, cylinder.

The authors would like to acknowledge the support from China Postdoctoral Science Foundation Grant 2013M540806, National Natural Science Foundation of China under grant 11271244 and National Natural Science Foundation of China under grant 11271282 and the 973 Program 2013CB834201. 
For some special classes of origin-symmetric convex bodies in $\mathbb{R}^{n}$, a sharper estimate for the lower bound of $\mathcal{P}(K)$ has been obtained. If $K$ is a convex body which is symmetric around all coordinate hyperplanes, Saint Raymond [24] proved that $\mathcal{P}(K) \geq 4^{n} / n$ !; the equality case was discussed in $[17,23]$. When $K$ is a zonoid (limits of finite Minkowski sums of line segments), Meyer and Reisner (see, e.g., $[9,21,22]$ ) proved that the same inequality holds, with equality if and only if $K$ is an $n$-cube. For the case of polytopes with at most $2 n+2$ vertices (or facets) (see, e.g., [2] for references), Lopez and Reisner [13] proved the inequality (1.1) for $n \leq 8$ and the minimal bodies are characterized. Recently, Nazarov, Petrov, Ryabogin and Zvavitch [20] proved that the cube is a strict local minimizer for the Mahler volume in the class of origin-symmetric convex bodies endowed with the Banach-Mazur distance.

Bourgain and Milman [3] proved that there exists a universal constant $c>0$ such that $\mathcal{P}(K) \geq c^{n} \mathcal{P}(B)$, which is now known as the reverse Santaló inequality. Very recently, Kuperberg [11] found a beautiful new approach to the reverse Santaló inequality. What's especially remarkable about Kuperberg's inequality is that it provides an explicit value for $c$.

Another variant of the Mahler conjecture without the assumption of originsymmetry states that, for any convex body $K$ in $\mathbb{R}^{n}$,

$$
\mathcal{P}(K) \geq \frac{(n+1)^{(n+1)}}{(n !)^{2}},
$$

with equality conjectured to hold only for simplices. For $n=2$, Mahler himself proved this inequality in 1939 (see, e.g., [5, 6, 14] for references) and Meyer [18] obtained the equality conditions in 1991. Recently, Meyer and Reisner [19] have proved inequality (1.2) for polytopes with at most $n+3$ vertices. Very recently, Kim and Reisner [10] proved that the simplex is a strict local minimum for the Mahler volume in the Banach-Mazur space of $n$-dimensional convex bodies.

Strong functional versions of the Blaschke-Santaló inequality and its reverse form have been studied recently (see, e.g., $[1,7]$ ).

The Mahler conjecture is still open even in the three-dimensional case. Terence Tao in [26] made an excellent remark about the open question.

In the following, we give the definition of parallel sections homothety bodies.

Definition 1. In $\mathbb{R}^{n}, u \in S^{n-1}$ and $L \subset\left\{x \in \mathbb{R}^{n}: x \cdot u=0\right\}$ is an originsymmetric convex body. Let $f(x)$ be a concave, even and nonnegative function defined on $[-a, a], a>0$. A parallel sections homothety body is defined as the convex body

$$
K=\bigcup_{x \in[-a, a]}\{f(x) L+x u\}
$$

where $f(x)$ is called its generating function and $L$ is its homothetic section.

In this paper, we prove that among some special parallel sections homothety bodies in $\mathbb{R}^{n}, n$-cubes have the minimal Mahler volume. 
Theorem 1.1. For a parallel sections homothety body $K$ in $\mathbb{R}^{n}$, if its homothetic section $L$ is a zonoid, then we have

$$
\mathcal{P}(K) \geq \frac{4^{n}}{n !}
$$

and the equality holds if and only if $L$ is an $(n-1)$-cube or an octahedron and its generating function $f(x)=f(0)$ or $f^{*}\left(x^{\prime}\right)=1 / f(0)$.

\section{Definitions, notation, and preliminaries}

As usual, $S^{n-1}$ denotes the unit sphere, and $B^{n}$ the unit ball centered at the origin, $O$ the origin and $\|\cdot\|$ the norm in Euclidean $n$-space $\mathbb{R}^{n}$. The symbol for the set of all natural numbers is $\mathbb{N}$. Let $\mathcal{K}^{n}$ denote the set of convex bodies (compact, convex subsets with non-empty interiors) in $\mathbb{R}^{n}$. Let $\mathcal{K}_{o}^{n}$ denote the subset of $\mathcal{K}^{n}$ that contains the origin in its interior. For $u \in S^{n-1}$, we denote by $u^{\perp}$ the $(n-1)$-dimensional subspace orthogonal to $u$. For $x, y \in \mathbb{R}^{n}, x \cdot y$ denotes the inner product of $x$ and $y$. We denote by $V(K)$ the $n$-dimensional volume of $K$.

If $K \in K_{o}^{n}$, we define the polar body $K^{*}$ of $K$ by

$$
K^{*}=\left\{x \in \mathbb{R}^{n}: x \cdot y \leq 1, \forall y \in K\right\} .
$$

For $K \in \mathcal{K}_{o}^{n}$, if $\left(x_{1}, x_{2}, \ldots, x_{n}\right) \in K$, we have $\left(\varepsilon_{1} x_{1}, \ldots, \varepsilon_{n} x_{n}\right) \in K$ for any signs $\varepsilon_{i}= \pm 1(i=1, \ldots, n)$, then $K$ is a 1 -unconditional convex body. In fact, $K$ is symmetric with respect to all coordinate planes.

For a convex body $K$ in $\mathbb{R}^{n}$, and $u \in S^{n-1}$, a Schwarz rounding of $K$ about the direction $u$ is any translate of the convex body $K$ for which for all $t \in \mathbb{R}$, $\tilde{K}(t)=\{x \in \tilde{K}: x \cdot u=t\}$, if it is not empty or a single point, is an $(n-1)$ dimensional Euclidean ball and $\operatorname{vol}_{n-1}(\tilde{K}(t))=\operatorname{vol}_{n-1}(K(t))$. In fact, the Schwarz rounding $\tilde{K}$ of $K$ is a special parallel sections homothety body, its homothetic section is a Euclidean ball.

\section{Main result and its proof}

Lemma 3.1. Let $f$ be a concave, even and nonnegative function defined on $[-a, a], a>0$. Then we have

$$
(n+1) \int_{0}^{a} f^{n}(t) d t \geq n f(x) \int_{0}^{a} f^{n-1}(t) d t+x f^{n}(0)
$$

or

$$
(n+1) \int_{0}^{a} f^{n}(t) d t \geq n f(x) \int_{0}^{a} f^{n-1}(t) d t+(a-x) f^{n}(a),
$$

for every $x \in[0, a]$ and every $n \geq 1$. 
Proof. By uniform approximation we may assume that $f$ is differentiable. By concavity we have for all $x, t \in[0, a], f(x) \leq f(t)+(x-t) f^{\prime}(t)$. Multiply both sides of the last inequality by $f(t)^{n-1}$ and integrate. This gives

$$
f(x) \int_{0}^{a} f^{n-1}(t) d t \leq \int_{0}^{a} f^{n}(t) d t+\frac{1}{n} \int_{0}^{a}(x-t)\left(f^{n}(t)\right)^{\prime} d t
$$

integration by parts gives

$$
\begin{aligned}
& \int_{0}^{a}(x-t)\left(f^{n}(t)\right)^{\prime} d t=(x-a) f^{n}(a)-x f^{n}(0)+\int_{0}^{a} f^{n}(t) d t \\
\leq & -x f^{n}(0)+\int_{0}^{a} f^{n}(t) d t, \quad\left(\text { or } \leq(x-a) f^{n}(a)+\int_{0}^{a} f^{n}(t) d t\right) .
\end{aligned}
$$

The last two inequalities taken together, prove the required inequality.

Lemma 3.2. Let $f$ be a concave, even and nonnegative function defined on $[-a, a], a>0$ and for $x^{\prime} \in\left[-\frac{1}{a}, \frac{1}{a}\right]$ define

$$
f^{*}\left(x^{\prime}\right)=\inf _{x \in[-a, a]} \frac{1-x^{\prime} x}{f(x)} .
$$

Then, for every integer $n \geq 0$

$$
\left(\int_{-a}^{a}(f(x))^{n} d x\right)\left(\int_{-1 / a}^{1 / a}\left(f^{*}\left(x^{\prime}\right)\right)^{n} d x^{\prime}\right) \geq \frac{4}{n+1} .
$$

Equality holds if and only if $f(x)=f(0)$ or $f^{*}\left(x^{\prime}\right)=1 / f(0)$.

Proof. We may assume that $f(0)=1$. For $n \geq 0$, we define the numbers $a_{n}$ and $b_{n}$ by $a_{n}=(n+1) \int_{0}^{a} f(x)^{n} d x$ and $b_{n}=(n+1) \int_{0}^{\frac{1}{a}}\left(f^{*}\left(x^{\prime}\right)\right)^{n} d x^{\prime}$. By Lemma 3.1. we have for $n \geq 1$ :

$$
\begin{gathered}
a_{n} \geq f(x) a_{n-1}+x \text { for every } x \in[0, a], \\
b_{n} \geq f^{*}\left(x^{\prime}\right) b_{n-1}+x^{\prime} \text { for every } x^{\prime} \in[0,1 / a] .
\end{gathered}
$$

It follows that $\frac{a_{n-1} f(x)}{a_{n}}+\frac{x}{a_{n}} \leq 1$ for every $x \in[0, a]$, which gives, by the definition of $f^{*}, f^{*}\left(\frac{1}{a_{n}}\right) \geq \frac{a_{n-1}}{a_{n}}$. Using the inequality (3.3), we get for every $n \geq 1, b_{n} a_{n} \geq a_{n-1} b_{n-1}+1$. By induction and $a_{0} b_{0}=1$, we get $a_{n} b_{n} \geq$ $a_{n-1} b_{n-1}+1 \geq a_{n-2} b_{n-2}+2 \geq \cdots \geq a_{0} b_{0}+n=n+1$. Thus, we have

$$
\left(\int_{-a}^{a}(f(x))^{n} d x\right)\left(\int_{-1 / a}^{1 / a}\left(f^{*}\left(x^{\prime}\right)\right)^{n} d x^{\prime}\right)=\frac{4 a_{n} b_{n}}{(n+1)^{2}} \geq \frac{4}{n+1} .
$$

The case of equality: It is easy to check that if $f$ satisfies the conditions at the end of the Lemma, equality holds. Suppose, on the other hand that we have equality. Then, we have $a_{1} b_{1}=a_{0} b_{0}+1=2$, which implies that $\int_{-a}^{a} f(x) d x \int_{-1 / a}^{1 / a} f^{*}\left(x^{\prime}\right) d x^{\prime}=2$. Let $C=\{(x, y): x \in[-a, a],|y| \leq f(x)\}$, then $C^{*}=\left\{\left(x^{\prime}, y^{\prime}\right): x^{\prime} \in[-1 / a, 1 / a],|y| \leq f^{*}\left(x^{\prime}\right)\right\}$, thus, $\mathcal{P}(C)=8$. Since the Mahler conjecture is correct for $n=2$, for any origin-symmetric convex body 
$K$ in $\mathbb{R}^{2}$, we have $\mathcal{P}(K) \geq 8$ with equality if and only if $K$ is a square or a diamond. Thus, $f$ satisfies the conditions at the end of the Lemma.

Lemma 3.3. For a parallel sections homothety body $K$ in $\mathbb{R}^{n}$, if

$$
K=\bigcup_{x \in[-a, a]}\{f(x) L+x u\},
$$

where $f(x)$ is its generating function and $L$ is its homothetic section. Then, we have

$$
K^{*}=\bigcup_{x^{\prime} \in[-1 / a, 1 / a]}\left\{f^{*}\left(x^{\prime}\right) L^{*}+x^{\prime} u\right\}
$$

where $f^{*}$ is given in (3.1).

Proof. Let

$$
K^{\prime}=\bigcup_{x^{\prime} \in[-1 / a, 1 / a]}\left\{f^{*}\left(x^{\prime}\right) L^{*}+x^{\prime} u\right\} .
$$

For any $v^{\prime} \in K^{\prime}$ and $v \in K$, there are $x^{\prime} \in[-1 / a, 1 / a], y^{\prime} \leq f^{*}\left(x^{\prime}\right), l^{\prime} \in L^{*}$, $x \in[-a, a], y \leq f(x)$ and $l \in L$ such that $v^{\prime}=y^{\prime} l^{\prime}+x^{\prime} u$ and $v=y l+x u$. Thus, we have

$$
\begin{aligned}
v^{\prime} \cdot v & =y^{\prime} y l^{\prime} \cdot l+x^{\prime} x \leq f^{*}\left(x^{\prime}\right) f(x) l^{\prime} \cdot l+x^{\prime} x \\
& \leq \frac{1-x^{\prime} x}{f(x)} f(x)+x^{\prime} x \leq 1
\end{aligned}
$$

which implies that $v^{\prime} \in K^{*}$.

On the other hand, if $v^{\prime}=y^{\prime} l^{\prime}+x^{\prime} u \notin K^{\prime}$, where $l^{\prime} \in L^{*}$, then either $\left|x^{\prime}\right|>1 / a$ or $\left|x^{\prime}\right| \leq 1 / a$ and $y^{\prime} l^{\prime} \notin f^{*}\left(x^{\prime}\right) L^{*}$. If $x^{\prime}>1 / a\left(\right.$ or $\left.x^{\prime}<-1 / a\right)$, then for $a u \in K($ or $-a u \in K)$, we have

$$
\left.v^{\prime} \cdot(a u)=x^{\prime} a>1\left(\text { or } v^{\prime} \cdot(-a u)\right)>1\right),
$$

which implies that $v^{\prime} \notin K^{*}$. If $\left|x^{\prime}\right| \leq 1 / a$ and $y^{\prime} l^{\prime} \notin f^{*}\left(x^{\prime}\right) L^{*}$, then there is $l \in L$ such that $y^{\prime} l^{\prime} \cdot l>f^{*}\left(x^{\prime}\right)$. Let $f^{*}\left(x^{\prime}\right)=\frac{1-x^{\prime} x_{0}}{f\left(x_{0}\right)}$. For $v_{0}=f\left(x_{0}\right) l+x_{0} u \in K$, we have

$$
\begin{aligned}
v^{\prime} \cdot v_{0} & =y^{\prime} f\left(x_{0}\right) l^{\prime} \cdot l+x^{\prime} x_{0}>f\left(x_{0}\right) f^{*}\left(x^{\prime}\right)+x^{\prime} x_{0} \\
& =f\left(x_{0}\right) \frac{1-x^{\prime} x_{0}}{f\left(x_{0}\right)}+x^{\prime} x_{0}=1,
\end{aligned}
$$

which implies that $v^{\prime} \notin K^{*}$. Hence, we have $K^{\prime}=K^{*}$.

In the following, we will restate and prove Theorem 1.1.

Theorem 3.4. For a parallel sections homothety body $K$ in $\mathbb{R}^{n}$, if its homothetic section is a zonoid, then we have $\mathcal{P}(K) \geq \frac{4^{n}}{n !}$, and the equality holds if and only if $L$ is an $(n-1)$-cube or an octahedron and its generating function $f(x)=f(0)$ or $f^{*}\left(x^{\prime}\right)=1 / f(0)$. 
Proof. Let

$$
K=\bigcup_{x \in[-a, a]}\{f(x) L+x u\}
$$

where $f(x)$ is its generating function and $L$ is its homothetic section. By $[9,22]$, if $L$ is a zonoid, by the known result, we have $\mathcal{P}(L) \geq \frac{4^{n-1}}{(n-1) !}$, with equality if and only if $L$ is an $(n-1)$-cube or an octahedron. Thus, by Lemma 3.3, we have $\mathcal{P}(K)=V(K) V\left(K^{*}\right)=\mathcal{P}(L) \int_{-a}^{a}(f(x))^{n-1} d x \int_{-\frac{1}{a}}^{\frac{1}{a}}\left(f^{*}\left(x^{\prime}\right)\right)^{n-1} d x^{\prime} \geq \frac{4^{n-1}}{(n-1) !} \frac{4}{n}=$ $\frac{4^{n}}{n !}$, the equality holds if and only if $L$ is an $(n-1)$-cube or an octahedron and its generating function $f(x)=f(0)$ or $f^{*}\left(x^{\prime}\right)=1 / f(0)$.

Remark 1. (1) In [9] and [22], the necessary and sufficient condition for equality to hold in inequality $\mathcal{P}(K) \geq \frac{4^{n}}{n !}$ is that $L$ is an $(n-1)$-cube, because that the polar body of a cube is an octahedron and the Mahler volumes of a convex body and its polar body are equivalent, therefore we can say that the necessary and sufficient condition for equality to hold is that $L$ is an $(n-1)$-cube or an octahedron.

(2) By Lemma 3.2 and Theorem 3.4, for any origin-symmetric convex body $K$ in $\mathbb{R}^{n}$, its Schwarz rounding $\tilde{K}$ satisfies $\mathcal{P}(\tilde{K}) \geq \frac{4}{n} \kappa_{n-1}^{2}$, where $\kappa_{n-1}=$ $V\left(B^{n-1}\right)$.

\section{References}

[1] S. Artstein, B. Klartag, and V. D. Milman, On the Santaló point of a function and a functional Santaló inequality, Mathematika 54 (2004), 33-48.

[2] K. Ball, Mahlers conjecture and wavelets, Discrete Comput. Geom. 13 (1995), no. 3-4, 271-277.

[3] J. Bourgain and V. D. Milman, New volume ratio properties for convex symmetric bodies in $\mathbb{R}^{n}$, Invent. Math. 88 (1987), no. 2, 319-340.

[4] S. Campi and P. Gronchi, Volume inequalities for $L_{p}$-zonotopes, Mathematika 53 (2006), no. $1,71-80$.

[5] _ On volume product inequalities for convex sets, Proc. Amer. Math. Soc. 134 (2006), no. 8, 2393-2402.

6] _ Extremal convex sets for Sylvester-Busemann type functionals, Appl. Anal. 85 (2006), no. 1-3, 129-141.

[7] M. Fradelizi, Y. Gordon, M. Meyer, and S. Reisner, The case of equality for an inverse Santaló functional inequality, Adv. Geom. 10 (2010), no. 4, 621-630.

[8] R. J. Gardner, Geometric Tomography, Second edition. Encyclopedia of Mathematics and its Applications, 58. Cambridge University Press, Cambridge, 2006.

[9] Y. Gordon, M. Meyer, and S. Reisner, Zonoids with minimal volume-product-a new proof, Proc. Amer. Math. Soc. 104 (1988), no. 1, 273-276.

[10] J. Kim and S. Reisner, Local minimality of the volume-product at the simplex, Mathematika, in press.

[11] G. Kuperberg, From the Mahler conjecture to Gauss linking integrals, Geom. Funct. Anal. 18 (2008), no. 3, 870-892.

[12] Y. Lin and G. Leng, Convex bodies with minimal volume product in $\mathbb{R}^{2}-a$ new proof, Discrete Math. 310 (2010), no. 21, 3018-3025. 
[13] M. A. Lopez and S. Reisner, A special case of Mahler's conjecture, Discrete Comput. Geom. 20 (1998), no. 2, 163-177.

[14] E. Lutwak, D. Yang, and G. Zhang, A volume inequality for polar bodies, J. Differential Geom. 84 (2010), no. 1, 163-178.

[15] K. Mahler, Ein Übertragungsprinzip für konvexe Körper, Casopis Pest. Mat. Fys. 68 (1939), 93-102.

[16] Ein Minimalproblem für konvexe Polygone, Mathematica (Zutphen) B. 7 (1939), 118-127.

[17] M. Meyer, Une caractérisation volumique de certains espacés normes de dimension finie, Israel J. Math. 55 (1986), no. 3, 317-326.

[18] Convex bodies with minimal volume product in $\mathbb{R}^{2}$, Monatsh. Math. 112 (1991), no. 4, 297-301.

[19] M. Meyer and S. Reisner, Shadow systems and volumes of polar convex bodies, Mathematika 53 (2006), no. 1, 129-148.

[20] F. Nazarov, F. Petrov, D. Ryabogin, and A. Zvavitch, A remark on the Mahler conjecture: local minimality of the unit cube, Duke Math. J. 154 (2010), no. 3, 419430 .

[21] S. Reisner, Random polytopes and the volume-product of symmetric convex bodies, Math. Scand. 57 (1985), no. 2, 386-392.

[22] _ Zonoids with minimal volume-product, Math. Z. 192 (1986), no. 3, 339-346.

[23] Minimal volume product in Banach spaces with a 1-unconditional basis, J. London Math. Soc. 36 (1987), no.1, 126-136.

[24] J. Saint-Raymond, Sur le volume des corps convexes sym etriques, Seminaire d'initiation 'al' Analyse, 1980/1981, Publ. Math. Univ. Pierre et Marie Curie, Paris, 1981, 1-25.

[25] R. Schneider, Convex Bodies: The Brunn-Minkowski Theory, Encyclopedia Math. Appl., vol. 44, Cambridge University Press, Cambridge, 1993.

[26] T. Tao, Structure and Randomness, Pages from year one of a mathematical blog. American Mathematical Society, Providence, RI, 2008.

YOUJIANG LIN

School of Mathematical SCiEnCES

PEKING UNIVERSITY

BeiJing, 100871, P. R. ChinA

AND

Department of Mathematics

Shanghai UNIVERSity

Shanghai, 200444, P. R. China

E-mail address: lxyoujiang@126.com

Gangsong LENG

Department of Mathematics

SHANGHAI UNIVERSITY

Shanghai, 200444, P. R. ChinA

E-mail address: gleng@staff.shu.edu.cn 